\title{
SHIMADZU: новинки аналитического и испытательного оборудования 2019
}

\author{
Т. Г. Борисова, А. В. Борисов, SHIMADZU Europa GmbH \\ smo_tb@shimadzu.ru
}

УДК 543.07; 620.17.051

Статья поступила в редакцию 25.07.2019

Статья принята к публикации 12.08.2019

\begin{abstract}
Компания SHIMADZU работает на аналитическом рынке более ста сорока лет и является одним из мировых лидеров по производству аналитического и испытательного оборудования. Выпускаемые приборы предназначены как для рутинного анализа, так и исследования сложных проб. Оборудование SHIMADZU используется в научноисследовательских лабораториях, аттестованных испытательных центрах, работающих в различных отраслях науки и производства: пищевой промышленности, фармацевтике, агропромышленном комплексе и др. Статья знакомит читателя с новыми приборами в области жидкостной хроматографии и испытательного оборудования, их возможностями и особенностями.
\end{abstract}

\section{ОБОРУДОВАНИЕ \\ ДЛЯ ЖИДКОСТНОЙ ХРОМАТОГРАФИИ}

Жидкостная хроматография занимает лидирующее положение среди методов анализа у химиков-аналитиков. Особенно популярны такие режимы работы, как традиционная и быстрая хроматография. Например, лаборатории пищевой промышленности обязаны ориентироваться на методы измерений, приведенные в нормативных документах (ГОСТ, МВИ и. Т.д.), в которых основную долю хроматографического анализа занимает традиционная жидкостная хроматография. В фармацевтике же наметился переход от классической хроматографии к быстрой и сверхбыстрой (СВЭЖХ, УВЭЖХ) как к более перспективным методам. Время анализа - один из ключевых показателей эффективности работы лаборатории, как при серийном производстве фармпрепаратов, так и проведении скрининговых исследований на стадиях разработки и клинических испытаний. Сверхбыстрая хроматография дает возможность сократить время анализа в несколько раз и повысить производительность лаборатории.

В 2019 году корпорация Shimadzu пополнила семейство блочных жидкостных хроматографов линейкой серии LC-40 Nexera (рис. 1). Новое оборудование предназначено для работы в режиме традиционной (44 МПа), быстрой (70 и 105 МПа) и сверхбыстрой (130 МПа) хроматографии и является пионером и эталоном инновационных технологий в жидкостной хроматографии с точки зрения эффективности, интеллектуального управления функциями прибора и дизайна.
Отличительная особенность жидкостных хроматографов - система искусственного интеллекта (IA), которая позволяет прибору самостоятельно обнаруживать и решать возникающие в ходе анализа проблемы. Состояние прибора и всех расходных деталей регистрируются на каждом этапе, данные от различных блоков системы собираются в облачном хранилище и анализируются для правильной настройки интервалов технического обслуживания. Это гарантия минимального простоя системы и максимальной экономии времени.

Хроматографическая система LC-40 Nexera способна отслеживать колебания давления подвижной фазы и изменения базовой линии. Пузырьки воздуха, образующиеся в подвижной фазе, могут вызвать проблемы при попадании в насос.

Когда система обнаруживает необычные колебания, она может автоматически очистить линии подачи подвижной фазы и повторно ввести образец сразу же после того, как поток и давление подвижной фазы восстановятся до нормальных показателей.

Для предотвращения повреждения колонок ВЭЖХ при запусках и остановках насоса или экстремальных изменениях градиента, в серии LC-40 Nexera реализована функция Smart Flow Pilot, которая постепенно увеличивает поток подвижной фазы до заданного значения. Таким образом, исключается необходимость создавать протоколы запуска для каждого анализа. 


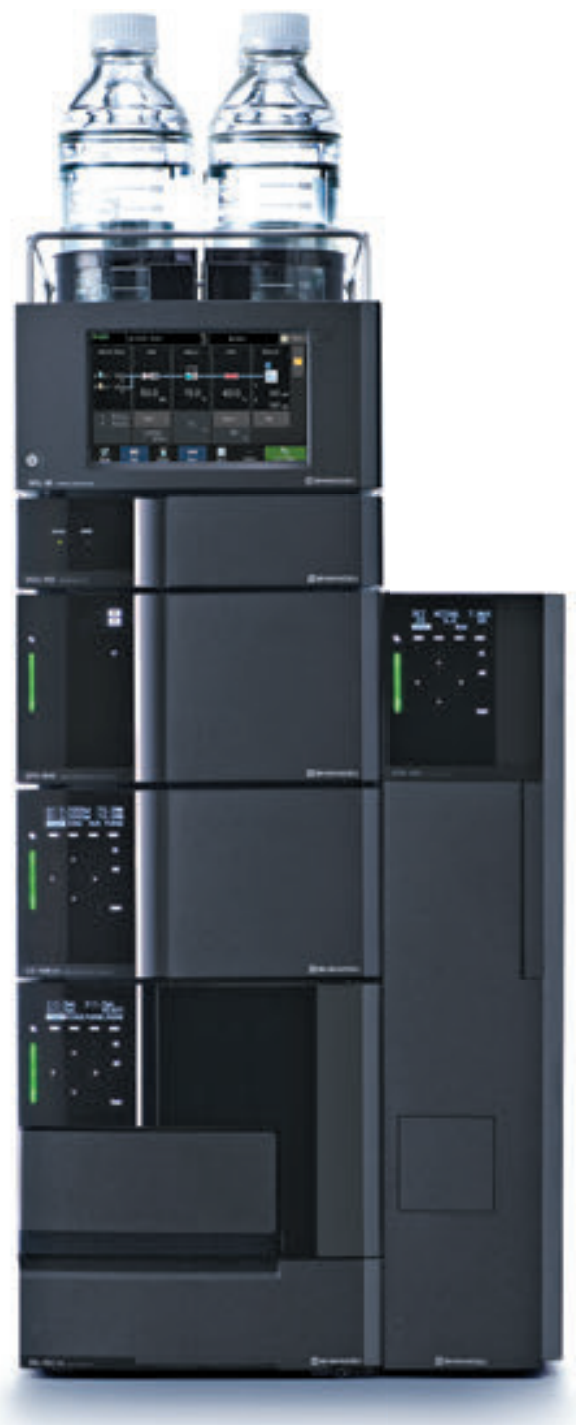

Puc. 1.

Жидкостный хроматограф серuи LC-40 Nexera

Системный контроллер SCL-40 имеет сенсорную панель и позволяет управлять прибором напрямую, без использования ПК.

Все модели автосамплеров 40-й серии имеют функцию автоматизированной подготовки образцов, которая включает разбавление, добавление внутренних стандартов и проведение дериватизации. Автоматизация подготовки образцов к анализу сокращает трудозатраты и исключает человеческий фактор. Ранее это было доступно только для автосамплера SIL-30AC серии Nexera X2 и моноблоков і-серии Plus.

Кроме того, реализована уникальная техника, позволяющая контролировать направление воздушного потока и предотвращать попадание окружающего воздуха в автосамплер (рис. 2). За счет прецизионного контроля температуры автодозатора и устройства автоматической загрузки пользователь может оперативно добавлять / заменять планшеты или подставки с виалами в ходе анализа.

Поддон для растворителей вмещает до 12 резервуаров (рис. 3) и оснащен датчиками веса для контроля количества подвижной фазы и растворов для промывки автодозатора.
Теперь не нужно заранее рассчитывать, хватит ли подвижной фазы для проведения анализа. Система это гарантирует, в противном случае пользователь получит предупреждение. Мониторинг количества подвижной фазы можно проводить удаленно с помощью соответствующего мобильного устройства.

Жидкостные хроматографы серии LC-40 Nexera могут быть укомплектованы спектрофотометрическими SPD-40/ SPD-40V детекторами, диодно-матричным SPD-M40, спектрофлуориметрическим RF-20A/RF-20Axs и диодноматричным SPD-M30A детекторами, спектрофотометрическими, диодно-матричным и рефрактометрическим детекторами серии Prominence, кондуктометрическим детектором 10-й серии, а также сверхбыстрыми жидкостными массспектрометрическими детекторами.

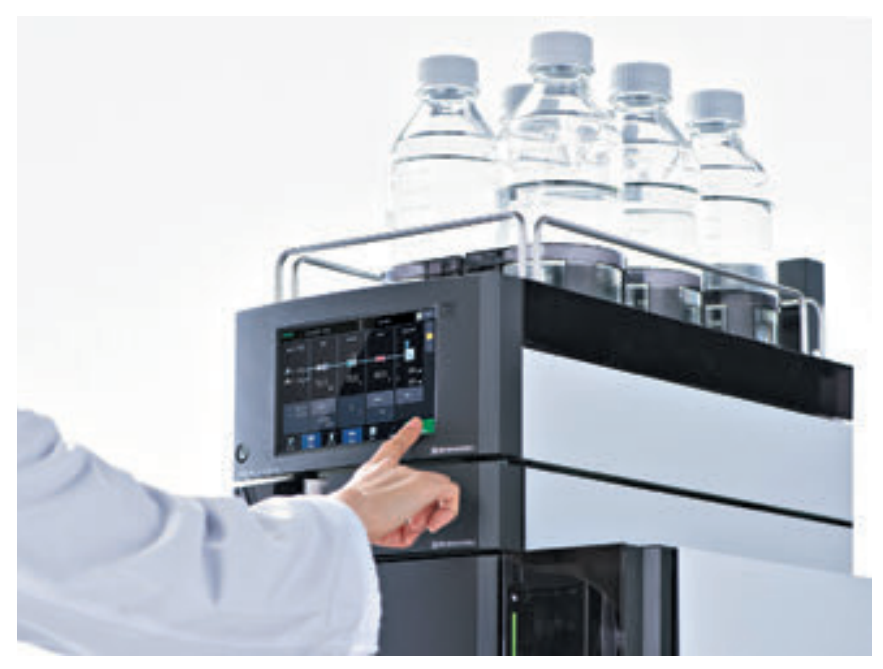

Puc. 3. Поддон для бутылей с растворителями, оснащенный датчиком веса 


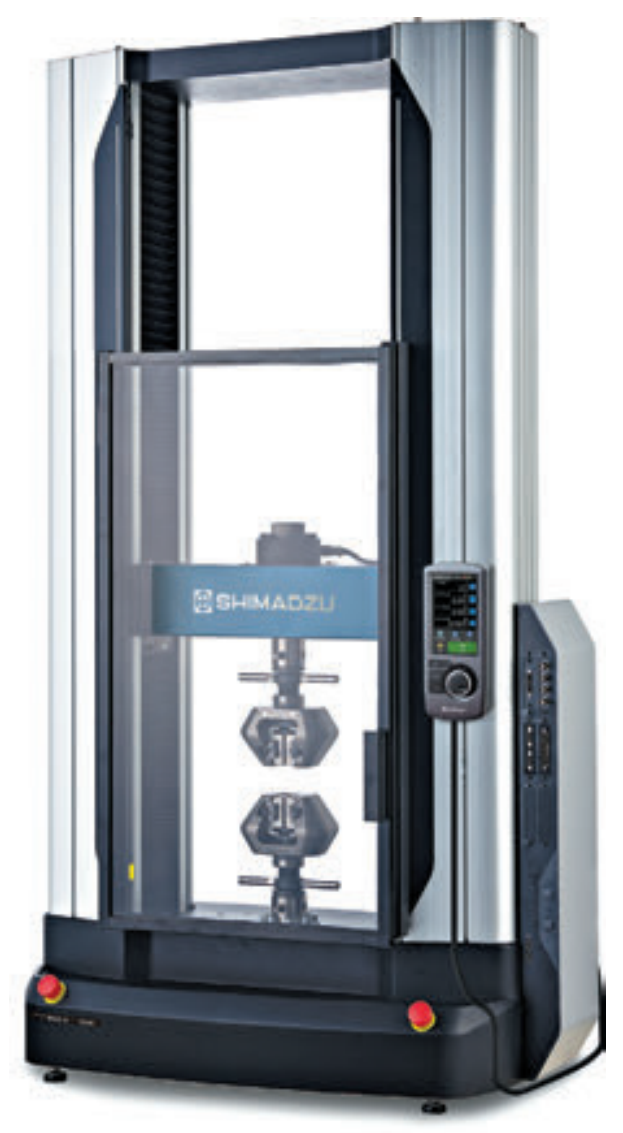

Puc. 4. Hacmonbная uспытательная машина серии AGX-V рассчитанная на максимальную нагрузку $50 \mathrm{kH}$

Габариты жидкостных хроматографов серии LC-40 Nexera уменьшены на две трети по сравнению с предыдущими моделями, что позволяет эффективнее использовать рабочее пространство лаборатории.

\section{ИСПЫТАТЕЛЬНОЕ ОБОРУДОВАНИЕ}

В 2019 году в сегменте испытательного оборудования компании SHIMADZU произошли изменения. Универсальные испытательные машины серии AG-Х получили развитие в новой линейке приборов - высокоточных машинах исследовательского класса серии AGX-V.

Практически все элементы подверглись модернизации: усовершенствованы силовая рама машины, контроллер, соединительные элементы, датчики нагрузки и даже программное обеспечение.

Для настольных моделей увеличена жесткость рамы, в том числе за счет нового корпуса колонн из экстрадированного алюминия. Специальные уплотнительные втулки колонн в нижней части корпуса машины также придают конструкции дополнительную жесткость. В корпусе предусмотрены Т-образные проточки по всей высоте машины для удобной установки дополнительной оснастки.

В модернизированный контроллер с усовершенствованной системой обработки данных можно установить до 20-ти усилителей для разного рода датчиков. Он позволяет контролировать положение траверсы с откликом 1 кГц и производить выборку данных с частотой до 10 кГц благодаря одновременной многопоточной обработке.

Новый выносной пульт управления оснащен сенсорным дисплеем, на котором в режиме реального времени отображается состояние системы, в том числе и информация о текущем и регламентированном расстоянии между захватами, и результаты испытания. Пульт управления полностью синхронизирован с пО.

Благодаря функции интеллектуального управления траверсой пользователь может задать необходимое расстояние между захватами. Машина будет автоматически занимать это положение при соответствующей команде. Кроме того, система постоянно отслеживает расстояние между захватами для предотвращения их столкновения.

Следующим закономерным шагом для повышения удобства и безопасности использования машины стал новый универсальный соединительный элемент. Установка дополнительного датчика нагрузки (без демонтажа основной ячейки), а также замена оснастки на растяжение, сжатие и изгиб теперь производятся при помощи легких небольших переходников.

Диапазон гарантированной точности датчика нагрузи стал шире и теперь начинается от 1/2000 его максимального номинального значения, благодаря чему минимальные нагрузки можно уверенно измерять на датчиках большого номинала. Таким образом, чтобы закрыть весь диапазон испытаний потребуется всего одна измерительная ячейка. Это не только упрощает работу с прибором, но и сокращает издержки на проведение процедур поверки оборудования.

Серия AGX-V - первые машины с заводской регулировкой соосности захватов, соответствующей классу 10 по ASTM E1012. Такая настройка машины обеспечивает высокую достоверность и повторяемость результатов, что особенно актуально для испытания высокопрочных материалов и композитов.

Новое программное обеспечение TRAPEZIUMX-V порадует пользователей не только приятным и удобным в работе интерфейсом, но и гибкостью, позволяющей оператору самостоятельно, не прилагая особых усилий, задать метод испытания согласно любому ГОСТ, ISO или ASTM. В интерфейсе ПО предусмотрено меню на русском языке.

$$
\because \because
$$

Жидкостный хроматограф серии LC-40 Nexera и другие приборы будут продемонстрированы на стенде компании на выставке "Химия-2019», они полностью отвечают запросам пользователей современного аналитического и испытательного оборудования. Оборудование SHIMADZU - это проверенные временем технологии, интуитивно понятное программное обеспечение с возможностью подключения, управления и сбора данных с различных типов приборов, а также простота в обслуживании и эксплуатации. 
$\oplus$ SHIMADZU Excellence in Science
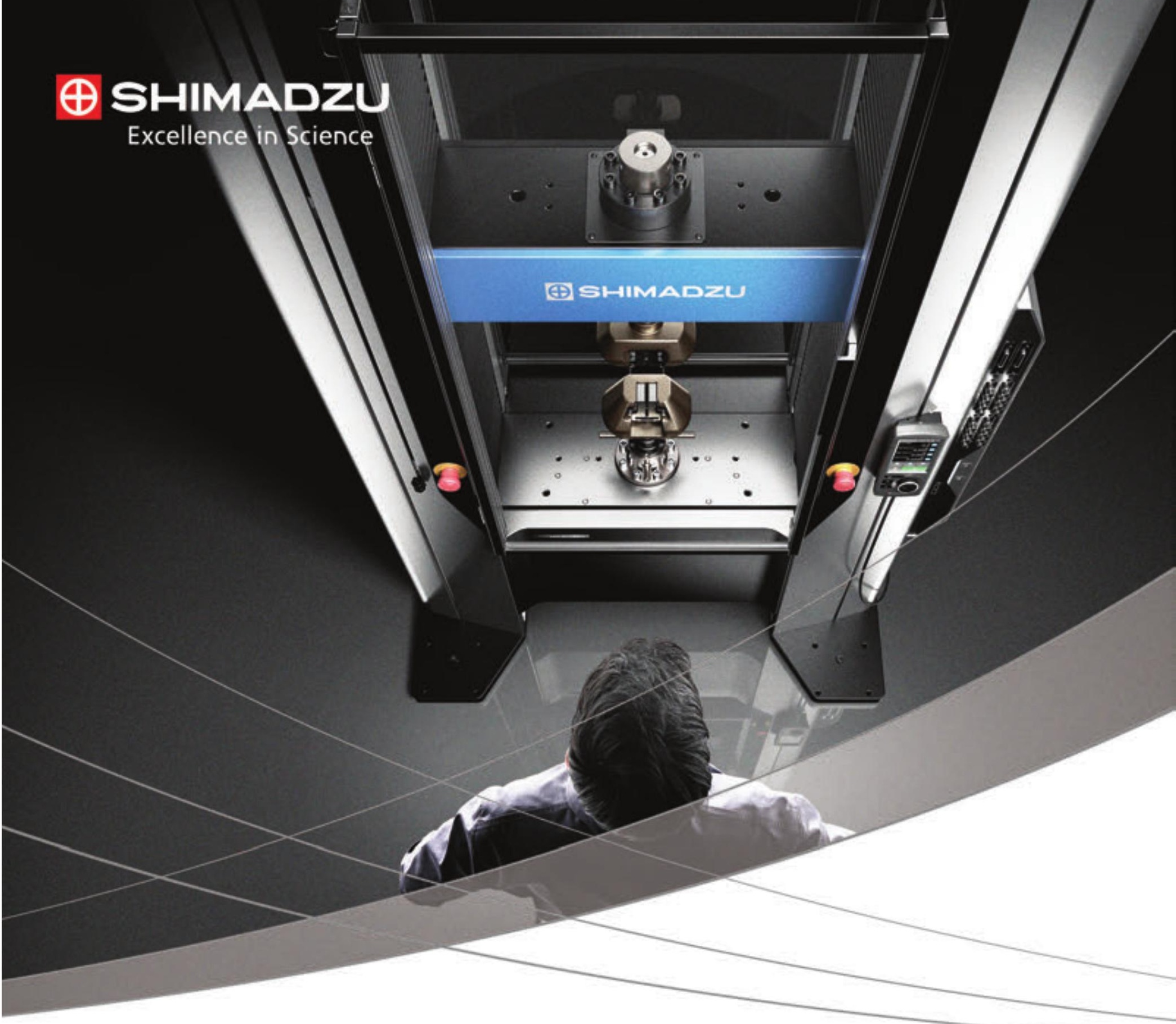

Высокоточные универсальные испытательные машины серии AGX-V

Корпорация Shimadzu занимается производством испытательного оборудования более 100 лет.

Серия AGX-V - новейшие испытательные машины в линейке универсальных машин AUTOGRAPH.

- Диапазон гарантированной точности от 1/2000 максимального номинала датчика

- Частота выборки данных до $10 \mathrm{kГц}$

- Интеллектуальная система управления траверсой

- Выносной сенсорный пульт управления

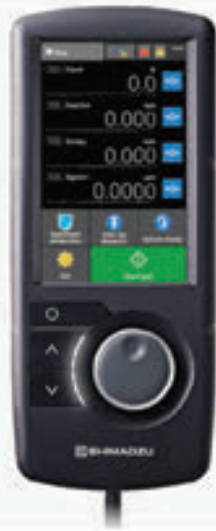

